\title{
玉米生长素响应因子家族基因的表达模式分析
}

李文兰李文才孙琦 于彦丽赵勐 鲁守平李艳娇 孟昭东*

山东省农业科学院玉米研究所 / 小麦玉米国家工程实验室 / 农业部黄淮海北部玉米生物学与遗传育种重点实验室, 山东济南 250100

摘 要: 生长素响应因子(auxin response factor, ARF)是一类重要的转录因子, 通过特异性地结合生长素响应元件调 节下游靶基因的转录, 参与诸多植物生长发育过程的调控。玉米中有许多 $A R F$ 家族基因, 但其表达模式有待深入研 究。本研究分析了玉米 $A R F$ 家族基因在不同组织器官中的表达, 发现除 $A R F 10 、 A R F 16$ 和 $A R F 34$ 组成型表达外, 其 余 32 个 $A R F$ 基因的表达水平在生殖器官中要明显高于营养器官。对 $A R F$ 基因启动子区的顺式作用元件分析显示, 28 个 $A R F$ 基因的启动子区含有逆境胁迫相关顺式元件, 实时定量 PCR 分析结果显示, 多个 $A R F$ 基因分别响应冷、热、 盐和渗透胁迫。研究结果不仅暗示了 $A R F$ 家族基因在玉米生殖生长和非生物逆境胁迫响应中的重要性, 也为全面解 析 $A R F$ 基因在玉米中的生物学功能提供有用信息。

关键词: $A R F$; 表达; 逆境胁迫; 玉米

\section{A study of expression pattern of auxin response factor family genes in maize (Zea mays L.)}

\author{
LI Wen-Lan, LI Wen-Cai, SUN Qi, YU Yan-Li, ZHAO Meng, LU Shou-Ping, LI Yan-Jiao, and MENG \\ Zhao-Dong*
}

Maize Research Institute, Shandong Academy of Agricultural Sciences / National Engineering Laboratory of Wheat and Maize / Key Laboratory of Biology and Genetic Improvement of Maize in Northern Yellow-Huai Rivers Plain, Ministry of Agriculture, Jinan 250100, Shandong, China

\begin{abstract}
Auxin response factors (ARFs) are important transcription factors which control the expression of target genes by binding specifically to auxin response elements, and are involved in a series of developmental processes in plant species. In maize genome, dozens of $A R F$ genes are encoded, however, there is little known on their expression patterns. In this study, the analysis on the expression level of $A R F$ genes in diverse tissues and organs revealed that expression level of $32 A R F$ genes were higher in reproductive organs than that in vegetative organs, except $A R F 10, A R F 16$, and $A R F 34$ constitutively expressed. The predicted results of $c i s$-acting elements showed that the promoter regions of $28 A R F$ genes harbored the cis-regulatory elements related to abiotic stresses. Real-time quantitative PCR results indicated that expression of several $A R F$ genes showed a response to cold, heat, and osmotic stresses, respectively. The results highlighted the importance of $A R F$ family genes in reproductive growth and abiotic stress response, and provided useful information for the comprehensive analysis of the biological function of $A R F$ genes in maize.
\end{abstract} Keywords: auxin response factor; expression; abiotic stress; maize (Zea mays L.)

生长素响应因子(auxin response factor, ARF)是 植物所特有的一类转录因子 ${ }^{[1]}$, 通过特异性地结合 生长素响应元件(AuxREs)调节下游靶基因的转录, 参与诸多植物生长发育过程的调控, 例如向性运 动、顶端优势、干旱胁迫响应等。研究 $A R F$ 基因的

\begin{abstract}
表达模式对于全面了解玉米 $A R F$ 基因家族的生物学 功能具有重要意义。

生长素信号转导过程涉及早期响应基因 ( AUX/IAA、GH3 和 SAUR 基因家族等)和与 AuxREs 相互作用的 $A R F$ 家族基因 ${ }^{[2]}$ 。 $\mathrm{ARF}$ 蛋白结合到生长
\end{abstract}


素调控基因启动子区的生长素响应元件 (5'-TGTCTC-3')上, 抑制或激活这些基因的转录 ${ }^{[3]}$, 从而影响植物的生长发育。拟南芥、水稻、二穗短 柄草、鹰嘴豆和丹参等多种植物中的 $A R F$ 基因已有 相关研究 ${ }^{[4-9]}$ 。拟南芥中第一个被发现的 $A R F$ 转录因 子是 $A R F 1$, 是以人工合成的生长素响应元件为诱饵, 通过活性很高的酵母单杂系统克隆得到的 ${ }^{[3]}$ 。ARF1 蛋白包括 665 个氨基酸, $\mathrm{N}$ 端包含 1 个 DNA 结合结 构域; 中间的一段区域富含脯氨酸, 丝氨酸和苏氨 酸含量也较高 ${ }^{[3]}$, 与其他富含脯氨酸结构域的转录 抑制子和转录激活子序列类似 ${ }^{[4]}$, 该区域可能是转 录抑制或转录激活结构域; C 端与 $A u x / I A A$ 基因编码 蛋白的结构域 III 和 IV 有类似结构 ${ }^{[3,10]}$ 。拟南芥中有 22 个基因编码 $\mathrm{ARF}$ 蛋白 ${ }^{[11]}$, 其中 $M P / A R F 5$ 参与胚 胎发生与维管形成 ${ }^{[12]} ; N P H 4 / A R F 7$ 参与植物向光性 和向重力性 ${ }^{[13]} ; A R F 19$ 控制叶扩展和侧根生长, 并 与 $N P H 4 / A R F 7$ 表现出功能几余性 ${ }^{[14-15]} ; A R F 6$ 和 $A R F 8$ 参与花成熟调控, 且呈现功能元余性 ${ }^{[16]}$ 。此外, 马铃薯 ARF6 参与顶端休眠, 调控与顶端分生组织 连接维管束的发育 ${ }^{[17]}$; 水稻 $O S A R F 1$ 受生长素诱导, 参与胚芽鞘的向性运动 ${ }^{[18]}$; 番茄 $D R 12$ 也是 $A R F$ 家 族基因, 参与种子发育、幼苗生长和果实成熟 ${ }^{[19]}$ 。

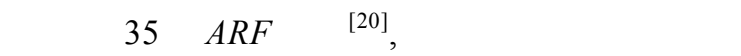
等已有相关分析, 但其表达模式还有待深入研究。 关于 $A R F$ 基因在玉米不同组织器官中的表达模式以 及在冷、热、盐和渗透胁迫下的表达模式还未见详 细报道。 Liu 等 ${ }^{[20]}$ 通过全基因组序列分析, 找到了 35 个玉米 $A R F$ 基因, 并对基因结构和进化关系等进 行了分析。本研究拟通过分析 $A R F$ 基因在玉米不同 组织器官中的表达模式以及在冷、热、盐和渗透胁 迫下的表达模式, 探究 $A R F$ 基因在玉米生长发育和 逆境胁迫响应过程中的作用, 为全面解析玉米 $A R F$ 基因的生物学功能提供有用信息。

\section{1 材料与方法}

\section{1 试验材料与生长条件}

试验所用的玉米材料是B73自交系。分别取材播 种后 $14 \mathrm{~d}$ (V3 时期)的初级根、第2片叶、整个幼苗植 株、播种后 $25 \mathrm{~d}$ (V5 时期)的中部茎秆和播种后 $34 \mathrm{~d}$ (V7时期)的整个幼嫩雄花序及雌花序, 用于实时定 量PCR分析。逆境胁迫试验所用的材料在光照培养 箱中进行长日照培养(16 h 光照 $/ 8 \mathrm{~h}$ 黑暗), 生长 $14 \mathrm{~d}$ 。 冷胁迫处理是将幼苗置于 $4^{\circ} \mathrm{C}$ 培养箱中进行处理,
热胁迫处理是将幼苗置于 $40^{\circ} \mathrm{C}$ 培养箱中进行处理, 盐胁迫处理是利用 $250 \mathrm{mmol} \mathrm{L} \mathrm{L}^{-1}$ 的 $\mathrm{NaCl}$ 对幼苗进行 处理, 渗透胁迫处理是利用 $15 \%$ PEG-6000对幼苗进 行处理。每种胁迫处理均在处理 $0 、 1 、 2 、 4$ 和 $8 \mathrm{~h}$ 后, 收集对照植株和处理植株的第2 片叶用于实时定量 PCR分析。

\subsection{RNA 提取和实时定量 PCR 分析}

利用天根的多糖多酚总 RNA 提取试剂盒提取 各样品的总 RNA, 按照反转录试剂盒说明书完成 cDNA 的合成和纯化。实时定量 PCR 分析利用 Bio-Rad CFX96 实时定量检测系统(Bio-Rad, Hercules, CA, USA), 引物序列见表 1, 18S rRNA 基因作为 内参基因, 根据 $2^{-\Delta \Delta \mathrm{CT}}$ 计算基因相对表达量。对于胁 迫处理下玉米幼苗的 qRT-PCR 分析, 将各处理 $0 \mathrm{~h}$ 各基因表达量设为 1 , 并以此对照计算其在其他时 间点的相对表达量。实验数据通过 SPSS18.0 软件进 行差异性分析。

\section{3 利用转录组数据对 $A R F$ 基因进行表达分析}

利用从 MaizeGDB 数据库获得的玉米 $A R F$ 基因的 ID 号在 Genevestigator 网站(http://www.genevestigator. $\mathrm{com} / \mathrm{gv} /$ )搜索 $A R F$ 基因在不同组织器官中的转录表 达数据, 然后利用 Genevestigator 网站的系统软件对 转录组表达数据进行分析, 获得 $A R F$ 基因在不同组 织器官中的表达模式热点分布图 ${ }^{[21]}$ 。

\section{4 启动子区顺式作用元件分析}

利用 Gramene 网站(http://ensembl.gramene.org/ Zea_mays/Info/Index)搜索确定玉米 $A R F$ 家族基因起 始密码子上游 $1500 \mathrm{bp}$ 的启动子区序列, 利用 PlantCare 数据库 (http://bioinformatics.psb.ugent.be/ webtools/plantcare/html/)的顺式作用元件分析工具, 对玉米 $A R F$ 家族基因启动子区序列进行顺式作用元 件分析 ${ }^{[22]}$ 。

\section{2 结果与分析}

2.1 玉米 $\boldsymbol{A R F}$ 家族基因在不同组织器官中的表 达分析

为探究生长素响应因子 $A R F$ 家族成员在玉米中 的表达模式，我们利用实时定量 PCR 方法对 $A R F$ 基 因在 6 种不同组织器官(根、茎、叶、幼苗、幼嫩雄 花序和幼嫩雌花序)中的表达量进行了检测(图 1)。 $A R F 10 、 A R F 16$ 和 $A R F 34$ 在玉米中组成型表达, $A R F 1$ 在茎、幼苗和雄花序中高表达, ARF28 在叶和雌花序 中高表达, 而剩余的 30 个基因均在雄花序和雌花序 
表 1 实时定量 PCR 的引物序列

Table 1 Primer sequences for real-time quantitative PCR

\begin{tabular}{|c|c|c|c|}
\hline & 基因号 & 引物名称 & 引物序列 \\
\hline Gene name & Gene accession & Primer name & Primer sequence $\left(5^{\prime}-3^{\prime}\right)$ \\
\hline \multirow{2}{*}{ ARF1 } & $\mathrm{Zm} 00001$ & $\operatorname{arf1-s}$ & CGTGTATATGTATCCTTCC \\
\hline & d030803 & arf1-a & ATTGTCTTCTGAGTACCA \\
\hline \multirow[t]{2}{*}{ ARF2 } & $\mathrm{Zm} 00001$ & $\operatorname{arf2-s}$ & AAACGACTCTGGGTATGT \\
\hline & d031064 & arf2-a & CTGAAGGACTTGTGTCTG \\
\hline \multirow[t]{2}{*}{ ARF3 } & $\mathrm{Zm} 00001$ & arf3-s & AACGGCATCTCTAACTAC \\
\hline & d031522 & arf3-a & ACGAATCTATGGAATTGAAC \\
\hline \multirow[t]{2}{*}{ ARF4 } & $\mathrm{Zm} 00001$ & arf4-s & AGTTCCGATGGCAGTGTT \\
\hline & d032683 & arf4-a & CGAGGAACCGATGCAGAT \\
\hline \multirow[t]{2}{*}{ ARF5 } & $\mathrm{Zm} 00001$ & arf5-s & CGCAACAACAACAGGCATG \\
\hline & d001879 & arf5-a & GGGCTAAAAAGGGACTGGTT \\
\hline \multirow[t]{2}{*}{ ARF6 } & $\mathrm{Zm} 00001$ & arf6-s & AATCTCAGCAGCAGTTAA \\
\hline & d001945 & arf6-a & CTTGGGACTCTTGGTTTA \\
\hline \multirow[t]{2}{*}{ ARF7 } & $\mathrm{Zm} 00001$ & $\operatorname{arf7-s}$ & CTAGTGACGCCCTGTACC \\
\hline & $\mathrm{d} 003601$ & $\operatorname{arf7-a}$ & AGTAATAGACACGCTCGC \\
\hline \multirow[t]{2}{*}{ ARF8 } & $\mathrm{Zm} 00001$ & arf8-s & GTAGTTGAAGTGGATAATTGTT \\
\hline & d041056 & arf8-a & TCTGGAGAAGGCTGATTA \\
\hline \multirow[t]{2}{*}{ ARF9 } & $\mathrm{Zm} 00001$ & $\operatorname{arf9-s}$ & TCTGAACCTCTGGTATCC \\
\hline & $\mathrm{d} 041056$ & arf9-a & AACAATTATCCACTTCAACTAC \\
\hline \multirow[t]{2}{*}{ ARF10 } & $\mathrm{Zm} 00001$ & $\operatorname{arf10-s}$ & TGAACTCGAAATCAGCTGC \\
\hline & d042267 & $\operatorname{arf10-a}$ & AАCTCCAACCTCCACTTGC \\
\hline \multirow[t]{2}{*}{ ARF11 } & $\mathrm{Zm} 00001 \mathrm{~d}$ & $\operatorname{arf11-s}$ & ATGTTACAGGGAATGGGAATG \\
\hline & 043431 & arf11-a & CAACAGTTTGAGGAGCAGACG \\
\hline \multirow[t]{2}{*}{ ARF12 } & $\mathrm{Zm00001d}$ & $\operatorname{arf12-s}$ & ATGGTAGACTTGATAGGA \\
\hline & 043922 & arf12-a & CATATTCACAGTTCCAGTA \\
\hline \multirow[t]{2}{*}{ ARF13 } & $\mathrm{Zm00001d}$ & $\operatorname{arf13-s}$ & CAAGGCAATCACAATCTG \\
\hline & 049295 & $\operatorname{arf13-a}$ & CTGTCTGTTCATCCCAAA \\
\hline \multirow[t]{2}{*}{ ARF14 } & $\mathrm{Zm00001d}$ & arf14-s & AATGACCGTTCTACTCCAATCA \\
\hline & 050781 & arf14-a & CTATCTCAATGCCAAACAATCT \\
\hline \multirow[t]{2}{*}{ ARF15 } & $\mathrm{Zm} 00001 \mathrm{~d}$ & $\operatorname{arf15-s}$ & ATGAGGTCTTCGCCAGGAT \\
\hline & 051172 & $\operatorname{arf15-a}$ & GGACTGTGTCAGCGTCTTG \\
\hline \multirow[t]{2}{*}{ ARF16 } & $\mathrm{Zm00001d}$ & arf16-s & AGAACATTGCTGATAGAT \\
\hline & 053819 & $\operatorname{arf16-a}$ & TTGTGTATGACCTTGAAT \\
\hline \multirow[t]{2}{*}{ ARF17 } & $\mathrm{Zm} 00001 \mathrm{~d}$ & $\operatorname{arf17-s}$ & CCGTATATCCAAGGGTTTTG \\
\hline & 014013 & arf17-a & ATGTGGGGTCTCTTTATGTCA \\
\hline \multirow[t]{2}{*}{ ARF18 } & $\mathrm{Zm00001d}$ & $\operatorname{arf18-s}$ & GCAGCAGATGGGGAAGCA \\
\hline & 014377 & $\operatorname{arf18-a}$ & AACTCGACCGAACCGACG \\
\hline \multirow[t]{2}{*}{ ARF19 } & $\mathrm{Zm00001d}$ & arf19-s & AGAGGACGGCGGCAAGAT \\
\hline & 014507 & arf19-a & TGCTCGCCCTCGGGTAGT \\
\hline \multirow[t]{2}{*}{ ARF20 } & $\mathrm{Zm00001d}$ & $\operatorname{arf} 20-\mathrm{s}$ & CCACCAATGAAGCAAGAA \\
\hline & 015243 & $\operatorname{arf} 20-\mathrm{a}$ & GATAGACAACATCTGACACAT \\
\hline \multirow[t]{2}{*}{ ARF21 } & $\mathrm{Zm} 00001 \mathrm{~d}$ & $\operatorname{arf} 21-\mathrm{s}$ & AACAGAACAGCATTCAGT \\
\hline & 014690 & $\operatorname{arf} 21-\mathrm{a}$ & TGATTCAGTGGAAGAGATG \\
\hline \multirow[t]{2}{*}{ ARF22 } & $\mathrm{Zm} 00001 \mathrm{~d}$ & $\operatorname{arf} 22-\mathrm{s}$ & GCTTTCCGCCAGCCTCA \\
\hline & 016838 & $\operatorname{arf} 22-\mathrm{a}$ & CCGGTGTCACCACCGATG \\
\hline
\end{tabular}




\begin{tabular}{|c|c|c|c|}
\hline $\begin{array}{c}\text { 基因名 } \\
\text { Gene name }\end{array}$ & $\begin{array}{c}\text { 基因号 } \\
\text { Gene accession }\end{array}$ & $\begin{array}{c}\text { 引物名称 } \\
\text { Primer name }\end{array}$ & $\begin{array}{c}\text { 引物序列 } \\
\text { Primer sequence }\left(5^{\prime}-3^{\prime}\right)\end{array}$ \\
\hline \multirow[t]{2}{*}{ ARF23 } & $\mathrm{Zm00001d}$ & $\operatorname{arf} 23-s$ & CTGAGAGGACGGTGAGCAA \\
\hline & 000358 & arf23-a & CGCGACAGCCGAGAGGT \\
\hline \multirow[t]{2}{*}{ ARF24 } & $\mathrm{Zm00001d}$ & $\operatorname{arf} 24-s$ & GCCACTTTCAAGTCAGATT \\
\hline & 036593 & arf24-a & TTGGATTGTGCTCTCAGA \\
\hline \multirow[t]{2}{*}{ ARF25 } & $\mathrm{Zm} 00001 \mathrm{~d}$ & $\operatorname{arf} 25-\mathrm{s}$ & СССТCTTCTGTTCTTATGTTT \\
\hline & 038698 & $\operatorname{arf} 25-\mathrm{a}$ & TACTTCTTCACGGTTGGT \\
\hline \multirow[t]{2}{*}{ ARF26 } & $\mathrm{Zm} 00001 \mathrm{~d}$ & arf26-s & GCATTCGCCCTCTTCTGTT \\
\hline & 038698 & $\operatorname{arf} 26-\mathrm{a}$ & AGGCTCGCTTCCATTTACA \\
\hline \multirow[t]{2}{*}{ ARF27 } & $\mathrm{Zm} 00001 \mathrm{~d}$ & $\operatorname{arf} 27-\mathrm{s}$ & TTCCATCTCAАTCCTCAT \\
\hline & 039006 & arf27-a & ТСТАТССТСТТТСТТАТТСАС \\
\hline \multirow[t]{2}{*}{ ARF28 } & GRMZM2 & $\operatorname{arf} 28-\mathrm{s}$ & TTATGGTTCCAATACAAGAA \\
\hline & G075715 & $\operatorname{arf} 28-a$ & СТCATTCCTATTCCTTAGC \\
\hline \multirow[t]{2}{*}{ ARF29 } & Zm00001d & $\operatorname{arf} 29-\mathrm{s}$ & TTCAAGATCAGGGTTCAG \\
\hline & 011953 & arf29-a & GATTCAACCGTCAGAGAA \\
\hline \multirow[t]{2}{*}{ ARF30 } & Zm00001d & $\operatorname{arf30-s}$ & CAAGTTCTTCAACATCAG \\
\hline & 045026 & $\operatorname{arf30-a}$ & ATCCTGTATTATGGTTCAA \\
\hline \multirow[t]{2}{*}{ ARF31 } & Zm00001d & $\operatorname{arf31-s}$ & ACTCGCTGGGAAGAGGGCT \\
\hline & 023659 & $\operatorname{arf31-a}$ & CCTTTTGTCTGCTTCACCAC \\
\hline \multirow[t]{2}{*}{ ARF32 } & Zm00001d & $\operatorname{arf32-s}$ & AGCTGGTGCGGGGCAAC \\
\hline & 025871 & $\operatorname{arf32-a}$ & CCTGCAAGGCCTCAATGAC \\
\hline \multirow[t]{2}{*}{ ARF33 } & $\mathrm{Zm} 00001 \mathrm{~d}$ & $\operatorname{arf33-s}$ & AGTTGAATGCTCTTGGTA \\
\hline & 026540 & arf33-a & GTGAATCTGTGCTTCTTG \\
\hline \multirow[t]{2}{*}{ ARF34 } & Zm00001d & arf34-s & ATGCTGGGTTGTTTGGTT \\
\hline & 026590 & arf34-a & GCGGCTAGAAAGTGGAAT \\
\hline \multirow[t]{2}{*}{ ARF35 } & $\mathrm{Zm00001d}$ & $\operatorname{arf35-s}$ & ATGATATTGGAGCAGATG \\
\hline & 026687 & arf35-a & AAGAGCATTATGGTGTTC \\
\hline \multirow[t]{2}{*}{$18 \mathrm{~S}$ rRNA } & & $18 \mathrm{~S}-\mathrm{s}$ & AAACGGCTACCACATCCAAG \\
\hline & & $18 \mathrm{~S}-\mathrm{a}$ & CCTCCAATGGATCCTCGTTA \\
\hline
\end{tabular}

中优势表达。从整体空间表达上看, 各基因的表达 量高低呈现生殖器官>营养器官。玉米花序发育涉及 小穗对原基、小穗原基、花器官原基等各类原基发 育起始过程, 在这些原基起始部位都存在生长素积 累和转运 ${ }^{[23]}$ 。作为生长素响应因子, $A R F$ 基因在雌花 序和雄花序中高水平表达, 暗示 $A R F$ 基因参与花序 发育调控过程。

2.2 转录组数据库中玉米 $\boldsymbol{A R F}$ 家族基因在不同 组织器官中的表达分析

为进一步了解玉米 $A R F$ 家族基因的表达模式, 我们利用 Genevestigator 数据库中的转录组数据对 $A R F$ 家族基因在更多不同组织器官 (41 个组织器官) 中的表达模式进行了分析, 共获得 31 个玉米 $A R F$ 基 因(未获得 $A R F 9$ 、ARF19、ARF 26 和 $A R F 28$ 这 4 个
基因的表达信息)的表达信息, 结果如图 2 所示。除了 花粉, ARF10、ARF16 和 $A R F 34$ 在所检测的其余组织 器官中均有表达, 这与实时定量 PCR 结果基本吻合。 $A R F 1$ 和 $A R F 2$ 在花药中优势表达, 这与定量结 果显示其在雄花序中优势表达是一致的。此外, 除 了 $A R F 1$ 和 $A R F 2$, 其余 29 个玉米 $A R F$ 基因在雌花 序发育 (pistil, ovary, ear, spikelet)和种子发育过程 (embryo, endosperm, embryo sac, embryo-surrounding region, basal endosperm transfer layer)中均有较高的 表达量。这与实时定量 PCR 结果显示 $A R F$ 基因在生 殖器官发育过程中优势表达也是一致的。然而, 与 定量结果显示 $A R F$ 基因在茎中低表达(除了 $A R F 1$ 和 $A R F 10$ )相反, 有 29 个 $A R F$ 基因(除 $A R F 2$ 和 $A R F 3$ ) 在茎中具有较高的表达水平。这可能是由于取材时 

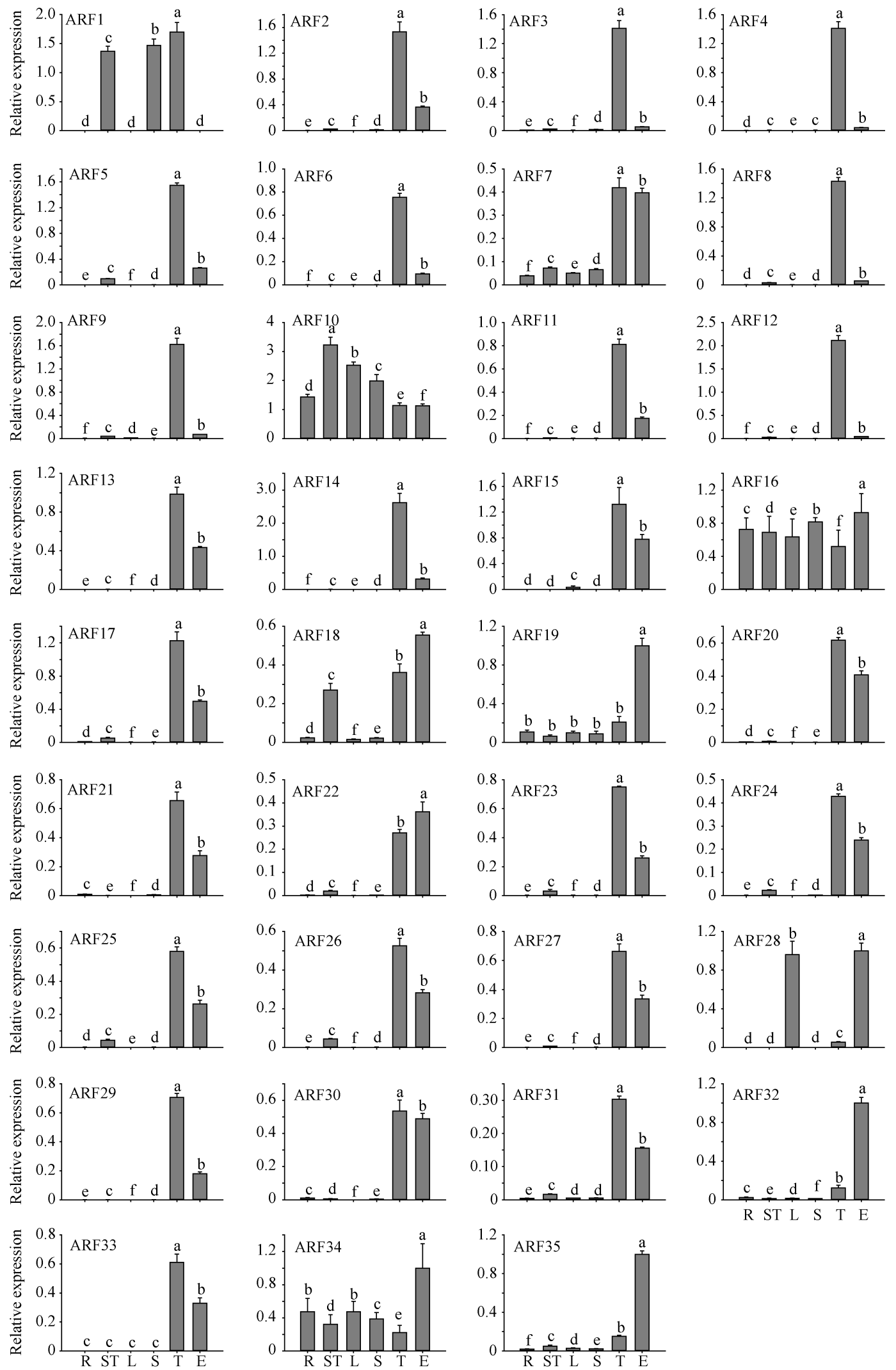

图 1 玉米 $A R F$ 家族基因在不同组织器官中的表达水平

Fig. 1 Expression patterns of $A R F$ genes in different tissues and organs

$\mathrm{R}$ : 根; ST: 茎; L: 叶; S: 幼苗; T: 雄花序; E：雌花序。图柱上的不同字母代表 Duncan's multiple range tests 的显著差异 $(P<0.05)$ 。

R: root; ST: stem; L: leaf; S: seedling; T: tassel; E: ear. Values marked with different letters indicate significant differences at $P<0.05$ by Duncan's multiple range tests. 


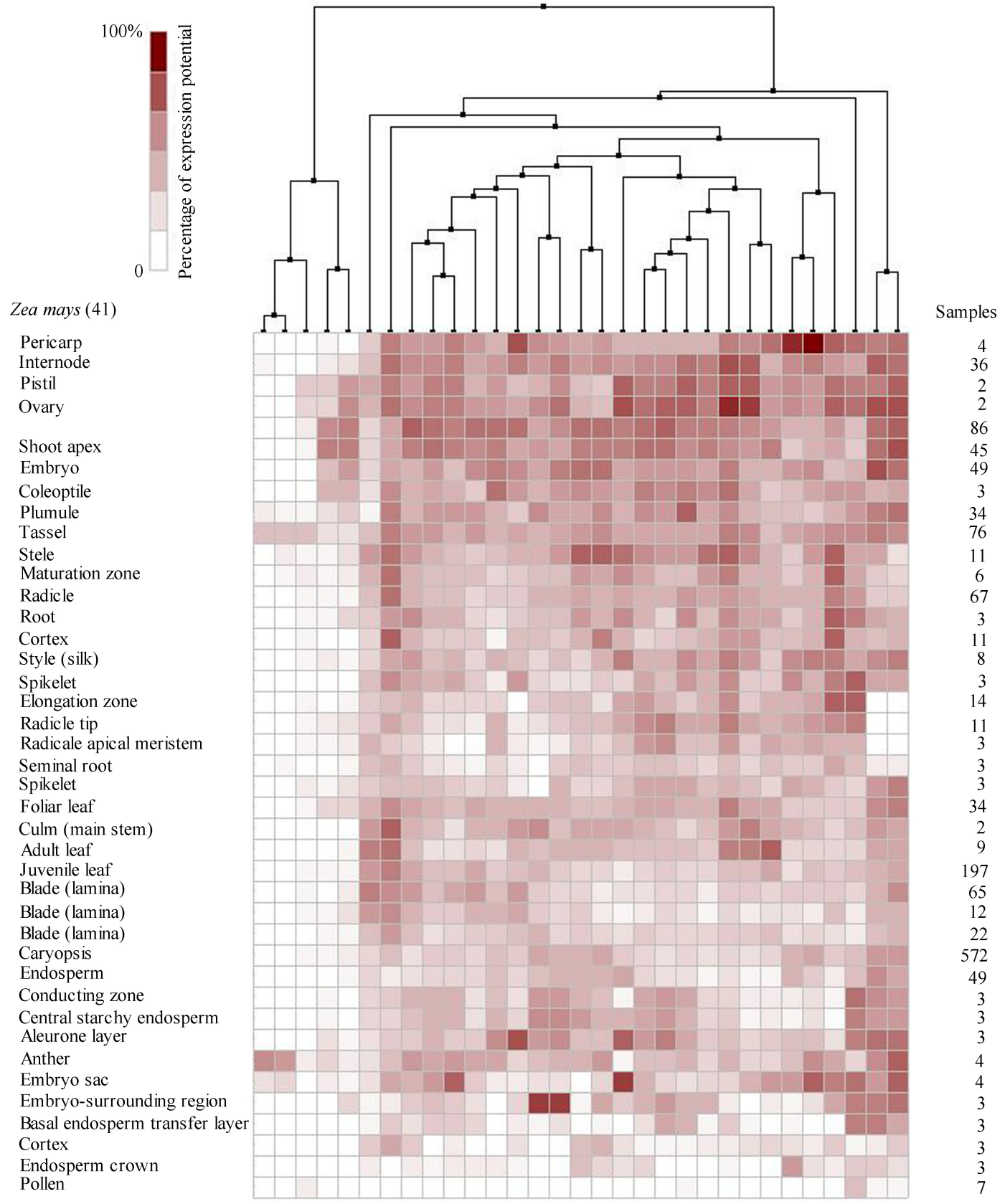

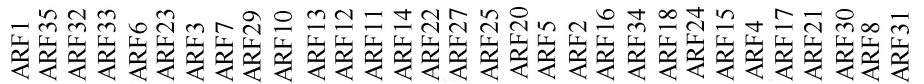

图 2 利用转录组数据获得的玉米 $\boldsymbol{A R F}$ 家族基因在不同组织器官中的表达水平

Fig. 2 Expression profile of $A R F$ genes in different tissues of maize by Genevestigator analysis 图中每个基因在所有检测组织器官中的表达量设为 $100 \%$, 颜色的深浅代表表达百分率。

The relative expression of each gene in all detected tissues and organs was set at $100 \%$, and the shade of color represents the percentage of relative expression levels.

期和试验材料不同所造成的结果差异。结合转录组 数据和实时定量 PCR 结果, 我们可以推测 $A R F$ 基因 在玉米生长发育和生殖器官发育过程中均发挥着重 要的作用。

\section{3 玉米 $A R F$ 家族基因的顺式作用元件分析} 为更好了解玉米 $A R F$ 家族基因在逆境胁迫响应
过程中的作用，我们对玉米 $A R F$ 家族基因起始密码 子上游 $1500 \mathrm{bp}$ 的启动子区进行顺式作用元件分析 (表2)，除转录和光响应相关必需顺式作用元件，在 玉米 $A R F$ 家族基因的启动子区找到大量顺式作用元 件，根据功能可分为：植物激素响应相关顺式作用 元件 (Element A)、生长发育相关顺式作用元件 
表 2 玉米 $\boldsymbol{A R F}$ 家族基因启动子区的顺式作用元件

Table 2 Putative cis-acting elements identified from the promoter regions of $A R F$ genes in maize

\begin{tabular}{|c|c|c|c|}
\hline $\begin{array}{l}\text { 基因 } \\
\text { Gene }\end{array}$ & $\begin{array}{c}\text { 元件 A } \\
\text { Element A } \\
\end{array}$ & $\begin{array}{c}\text { 元件 B } \\
\text { Element B }\end{array}$ & $\begin{array}{c}\text { 元件 C } \\
\text { Element C }\end{array}$ \\
\hline$A R F 1$ & $\begin{array}{l}\text { ABRE }^{1}, \text { AuxRR-core }^{1}, \text { CGTCA-motif }^{2}, \text { P-box }^{2}, \\
\text { TGA-element }^{2}, \text { TGACG-motif }\end{array}$ & & $\mathrm{MBS}^{1}$ \\
\hline$A R F 2$ & ABRE $^{3}$, CGTCA-motif $^{3}$, P-box ${ }^{1}$, TGACG-motif ${ }^{3}$ & CAT-box ${ }^{2}$ & \\
\hline$A R F 3$ & ABRE $^{3}$, CGTCA-motif $^{4}$, GARE-motif $^{1}$, P-box $^{1}$, TGACG-motif $^{4}$ & CAT-box ${ }^{2}$ & TCA-element ${ }^{2}$, LTR $^{1}$ \\
\hline ARF4 & ABRE $^{1}$, CGTCA-motif $^{1}$, P-box $^{1}$, TGACG-motif $^{1}$ & GCN4_motif ${ }^{1}$ & $\mathrm{LTR}^{1}, \mathrm{MBS}^{1}$ \\
\hline$A R F 5$ & ABRE $^{1}$, CGTCA-motif $^{1}$, GARE-motif $^{1}$, TGA-element $^{1}$, TGACG-motif $^{1}$ & CAT-box ${ }^{3}$ & TCA-element ${ }^{1}$, LTR $^{2}$ \\
\hline ARF6 & ABRE $^{2}$, CGTCA-motif $^{1}$, TGA-element $^{1}$, TGACG-motif $^{1}$ & CAT-box ${ }^{2}$, RY-element ${ }^{1}$ & $\mathrm{MBS}^{1}$ \\
\hline$A R F 7$ & ABRE $^{2}$, CGTCA-motif $^{1}$, TGA-element $^{1}$, TGACG-motif $^{1}$ & CAT-box $^{1}$ & $\mathrm{LTR}^{3}, \mathrm{MBS}^{1}$ \\
\hline$A R F 8$ & ABRE $^{2}$, CGTCA-motif $^{3}$, GARE-motif $^{1}$, TGACG-motif $^{3}$ & & LTR $^{1}$, MBS $^{1}$, TC-rich repeats ${ }^{1}$ \\
\hline$A R F 9$ & ABRE $^{2}$, CGTCA-motif $^{3}$, GARE-motif $^{1}$, TGACG-motif $^{3}$ & & LTR $^{1}$, MBS $^{1}$, TC-rich repeats ${ }^{1}$ \\
\hline ARF10 & ABRE $^{1}$, CGTCA-motif $^{5}$, TGACG-motif $^{5}$ & & \\
\hline ARF11 & ABRE $^{2}$, CGTCA-motif $^{2}$, TGA-element $^{1}$, TGACG-motif $^{2}$ & CAT-box ${ }^{2}$ & TCA-element ${ }^{1}$ \\
\hline$A R F 12$ & ABRE $^{1}$, CGTCA-motif $^{1}$, TGACG-motif $^{1}$ & CAT-box ${ }^{1}$ & $\mathrm{MBS}^{2}$ \\
\hline$A R F 13$ & ABRE $^{2}$, CGTCA-motif $^{2}$, P-box $^{1}$, TGACG-motif $^{2}$ & & TC-rich repeats ${ }^{1}$ \\
\hline ARF14 & $\mathrm{ABRE}^{2}, \mathrm{TGA}^{-e l e m e n t}{ }^{1}$ & & TCA-element ${ }^{2}$ \\
\hline$A R F 15$ & CGTCA-motif $^{3}$, TGA-element $^{1}$, TGACG-motif $^{3}$ & & $\mathrm{LTR}^{1}, \mathrm{MBS}^{1}$ \\
\hline ARF16 & ABRE $^{1}$, AuxRR-core ${ }^{1}$, CGTCA-motif $^{2}$, TGACG-motif $^{2}$ & CAT-box ${ }^{3}$ & \\
\hline$A R F 17$ & ABRE $^{1}$, CGTCA-motif $^{4}$, TGA-element $^{1}$, TGACG-motif $^{4}$ & RY-element ${ }^{1}$ & $\mathrm{MBS}^{1}, \mathrm{TCA}^{- \text {element }}{ }^{1}$ \\
\hline ARF18 & ABRE $^{2}$, CGTCA-motif $^{1}$, P-box $^{1}$, TGACG-motif $^{1}$ & & $\mathrm{MBS}^{1}$ \\
\hline ARF19 & P-box ${ }^{1}$ & CAT-box ${ }^{1}$ & LTR $^{1}$, MBS $^{1}$, TCA-element ${ }^{1}$ \\
\hline ARF20 & ABRE $^{3}$, CGTCA-motif $^{1}$, TGA-element $^{1}$, TGACG-motif $^{1}$ & & LTR $^{1}$, TCA-element ${ }^{1}$ \\
\hline$A R F 21$ & $\mathrm{ABRE}^{7}, \mathrm{CGTCA}^{-m_{0}}$ if $^{2}, \mathrm{TGACG}^{-m_{0}} \mathrm{tif}^{2}$ & & $\mathrm{MBS}^{1}$ \\
\hline$A R F 22$ & ABRE $^{6}$, CGTCA-motif $^{2}$, GARE-motif $^{1}$, TGACG-motif $^{2}$ & CAT-box $^{1}$ & \\
\hline$A R F 23$ & $\mathrm{ABRE}^{4}$, AuxRR-core $^{1}, \mathrm{CGTCA}^{-m o t i f}{ }^{1}$, TGA-element $^{2}$, TGACG-motif $^{1}$ & CAT-box $^{1}$ & LTR $^{1}$, MBS $^{2}$, TCA-element ${ }^{1}$ \\
\hline$A R F 24$ & ABRE $^{3}$, CGTCA-motif $^{2}$, GARE-motif $^{1}$, P-box $^{2}$, TGACG-motif $^{2}$ & & MBS $^{1}$, TCA-element $^{1}$ \\
\hline$A R F 25$ & ABRE $^{1}$, CGTCA-motif $^{1}$, P-box $^{1}$, TGACG-motif $^{1}$ & CAT-box $^{3}$ & LTR $^{1}$, TC-rich repeats $^{1}$ \\
\hline$A R F 26$ & ABRE $^{1}$, CGTCA-motif $^{1}$, P-box $^{1}$, TGACG-motif $^{1}$ & CAT-box ${ }^{3}$ & LTR $^{1}$, TC-rich repeats $^{1}$ \\
\hline$A R F 27$ & $\mathrm{ABRE}^{4}, \mathrm{GARE}^{-m o t i f}{ }^{1}$ & RY-element ${ }^{1}$ & \\
\hline$A R F 28$ & ABRE $^{1}$, CGTCA-motif $^{2}$, GARE-motif $^{1}$, TGACG-motif $^{2}$ & CAT-box ${ }^{2}$ & $\mathrm{MBS}^{2}$, TCA-element ${ }^{2}$ \\
\hline$A R F 29$ & ABRE $^{3}$, AuxRR-core $^{1}$, CGTCA-motif $^{1}$, TGACG-motif $^{1}$ & CAT-box $^{1}$ & $\mathrm{LTR}^{1}$ \\
\hline ARF30 & ABRE $^{6}$, CGTCA-motif $^{4}$, TGACG-motif $^{4}$ & circadian $^{1}$ & $\mathrm{MBS}^{2}$, TC-rich repeats ${ }^{1}$ \\
\hline$A R F 31$ & ABRE $^{1}$, CGTCA-motif $^{2}$, TGACG-motif $^{2}$ & circadian $^{1}$ & $\mathrm{LTR}^{2}$ \\
\hline$A R F 32$ & ABRE $^{5}$, CGTCA-motif $^{1}$, TGACG-motif $^{1}$ & $\begin{array}{l}\text { CAT-box }{ }^{1}, \text { GCN4_motif } \\
\text { RY-element }\end{array}$ & TCA-element ${ }^{1}$ \\
\hline ARF33 & $\mathrm{ABRE}^{4}, \mathrm{CGTCA}^{-m o t i f}{ }^{3}$, GARE-motif $^{1}$, TGA-element $^{1}$, TGACG-motif $^{3}$ & CAT-box ${ }^{4}$, RY-element ${ }^{1}$ & \\
\hline ARF34 & ABRE $^{1}$, GARE-motif $^{1}$, TGA-element $^{1}$ & CAT-box ${ }^{2}$ & $\operatorname{LTR}^{1}$ \\
\hline$A R F 35$ & $\mathrm{ABRE}^{3}$ & & $\mathrm{MBS}^{1}$ \\
\hline
\end{tabular}

ABRE: 脱落酸响应元件; AuxRR-core 和 TGA-element: 生长素响应元件; CGTCA-motif 和 TGACG-motif: 茉莉酸甲酯响应元件; GARE-motif 和 P-box：赤霉素响应元件; CAT-box：分生组织相关响应元件; circadian：生物钟相关响应元件; GCN4_motif: 胚乳表达相 关元件;RY-element: 种子特异调控响应元件; LTR: 低温响应元件; MBS: 干旱胁迫响应元件; TC-rich repeats: 防御胁迫响应元件; TCA-element: 水杨酸响应元件。肩标数字代表每个顺式作用元件在 $A R F$ 基因启动区的拷贝数。

ABRE: ABA-responsive element; LTR: low temperature-responsive element; MBS: MYB binding site involved in drought-inducibility. The shoulder number represents the number of copies of each cis-acting element in the $A R F$ gene promoter region. 
(Element B)和逆境胁迫相关顺式作用元件(Element C)。Element A中的脱落酸响应元件(ABRE)分布于除 $A R F 15$ 和 ARF19之外的每一个 $A R F$ 基因启动子区, 茉莉酸甲酯响应元件(CGTCA-motif, TGACG-motif, MeJA-responsive element)分布在除ARF14、ARF19、 $A R F 27 、 A R F 34$ 和 ARF35之外的30个 $A R F$ 基因启动子 区, 赤霉素响应元件 P-box (gibberellin-responsive element)和GARE-motif (gibberellin-responsive element) 分别位于 $A R F 1 、 A R F 2 、 A R F 3 、 A R F 4 、 A R F 13 、 A R F 18$ 、 $A R F 19 、 A R F 24 、 A R F 25 、 A R F 26$ 和 $A R F 3 、 A R F 5$ 、 $A R F 8 、 A R F 9 、 A R F 22 、 A R F 24 、 A R F 27 、 A R F 28 、$ $A R F 33 、 A R F 34$ 的启动子区, 生长素响应元件 AuxRR-core和TGA-element分别位于 $A R F 1 、 A R F 16$ 、 $A R F 23 、 A R F 29$ 和 $A R F 1 、 A R F 5 、 A R F 6 、 A R F 7 、 A R F 11$ 、 $A R F 14 、 A R F 15 、 A R F 17 、 A R F 20 、 A R F 23 、 A R F 33$ 和 $A R F 34$ 的启动子区。 $\mathrm{Xing}$ 等 ${ }^{[24]}$ 分析了 31 个 $A R F$ 基 因启动子区的生长素响应元件, 与本文结果基本吻 合。Element B中的分生组织相关响应元件(CAT-box) 位于 $A R F 2 、 A R F 3 、 A R F 5 、 A R F 6 、 A R F 7 、 A R F 11$ 、 $A R F 12 、 A R F 16 、 A R F 19 、 A R F 22 、 A R F 23 、 A R F 25 、$ $A R F 26 、 A R F 28 、 A R F 29 、 A R F 32 、 A R F 33$ 和 $A R F 34$ 的启动子区, 种子特异调控响应元件(RY-element)分 布在ARF6、ARF17、ARF27、ARF32和 $A R F 33$ 的启动 子区, 生物钟相关响应元件 (circadian) 只分布在 $A R F 30$ 和 $A R F 31$ 的启动子区, 胚乳表达相关元件 (GCN4_motif)只分布在 $A R F 4$ 和 $A R F 32$ 的启动子区。

Element $\mathrm{C}$ 中的低温响应元件 (low temperatureresponsive element, LTR)位于 $A R F 3 、 A R F 4 、 A R F 5$ 、 $A R F 7 、 A R F 8 、 A R F 9 、 A R F 15 、 A R F 19 、 A R F 20 、 A R F 23 、$ $A R F 25 、 A R F 26 、 A R F 29 、 A R F 31$ 和 $A R F 34$ 的启动子 区，干旱胁迫响应元件(MYB binding site involved in drought-inducibility, MBS)位于 $A R F 1 、 A R F 4 、 A R F 6$ 、 $A R F 7 、 A R F 8 、 A R F 9 、 A R F 12 、 A R F 15 、 A R F 17 、 A R F 18$ 、 $A R F 19 、 A R F 21 、 A R F 23 、 A R F 24 、 A R F 28 、 A R F 30$ 和 $A R F 35$ 的启动子区, 防御胁迫响应元件(TC-rich repeats)位于 $A R F 8 、 A R F 9 、 A R F 13 、 A R F 25 、 A R F 26$ 和 $A R F 30$ 的启动子区, 水杨酸响应元件 (TCA-element)位于 $A R F 3 、 A R F 5 、 A R F 11 、 A R F 14$ 、 $A R F 17 、 A R F 19 、 A R F 20 、 A R F 23 、 A R F 24 、 A R F 28$ 和 $A R F 32$ 的启动子区。其中, 植物激素响应相关顺式 作用元件最多, 每个 $A R F$ 基因都含有 $2 \sim 15$ 个; 生长 发育相关顺式作用元件分布在 23 个 $A R F$ 基因的启动 子区, 主要包括RY-element、GCN4-motif和circadian
等; 逆境胁迫相关顺式作用元件主要分布在 29 个 $A R F$ 基因的启动子区，主要包括MBS、LTR和TC-rich repeats等。每个 $A R F$ 基因的启动子区都包含多个顺式 作用元件, 表明玉米 $A R F$ 基因在植物激素响应、生长 发育和逆境胁迫响应等方面均发挥重要作用。

2.4 玉米 $\boldsymbol{A R F}$ 家族基因在冷、热、盐和渗透胁 迫条件下的表达分析

顺式作用元件分析显示有29个玉米 $A R F$ 基因启 动子区包含逆境胁迫相关顺式作用元件。为进一步 研究玉米 $A R F$ 家族基因在逆境胁迫响应过程中的作 用，我们选取了9个在叶片中表达量较高的 $A R F$ 基因 (ARF7、ARF10、ARF15、ARF16、ARF18、ARF19、 $A R F 32 、 A R F 34$ 和 $A R F 35)$, 利用实时定量苂光PCR方 法检测玉米 $A R F$ 家族基因在冷、热、盐及渗透胁迫 下的表达量变化(图3)。在冷胁迫处理下，所有被检 测 $A R F$ 基因的表达模式都是类似的，在处理 $1 \mathrm{~h}$ 时表 达量迅速上升, 随后降到较低水平, 处理4 h后又开 始上升。在热胁迫处理下, $A R F 16$ 基因 $1 \mathrm{~h}$ 时表达量达 到峰值, 是未处理时的 25 倍, 随后又逐渐下降, 而 其余 8 个 $A R F$ 基因的表达量则是随着时间逐渐上升。 在盐胁迫处理下, 多数 $A R F$ 基因的表达量变化不大 (除了 $A R F 10$ 在处理 $4 \mathrm{~h}$ 时表达量达到峰值随后又开 始下降外), 比较有趣的一点是, ARF35的表达量在 处理后迅速下降并维持在较低水平，暗示该基因可 能是通过与其他 $A R F$ 基因相反的途径参与盐胁迫调 控过程。在渗透胁迫处理下, $A R F 15 、 A R F 18$ 和 $A R F 19$ 的表达量是先上升后下降, 在处理 $8 \mathrm{~h}$ 时表达量达到 峰值; ARF 10、ARF32、ARF34和 $A R F 35$ 的表达模式 是相似的, 处理 $1 \mathrm{~h}$ 时表达量达到峰值, 随后下降并 维持在较低水平; $A R F 7$ 和 $A R F 16$ 的表达量变化不大。 这些结果表明, $A R F$ 基因涉及参与多种逆境胁迫响 应过程。

\section{3 讨论}

作为重要的植物激素, 生长素几乎参与了植物 生长发育的每个阶段。而 $A R F$ 基因作为生长素响应 因子, 通过特异性地结合生长素响应元件调节下游 靶基因的转录, 参与诸多植物生长发育过程 ${ }^{[2]}$ 。根据 标记基因表达情况可将 $A R F$ 家族分为 3 类：一类 (Clade A)具有转录激活功能, 一类(Clade B) 具有转 录抑制功能, 一类(Clade C)既不具有转录激活功能 也不具有转录抑制功能 ${ }^{[25]}$ 。这3 类 $A R F$ 基因的功能分 类与进化关系是一致的 ${ }^{[26]}$ 。结合不同数据库分析得 

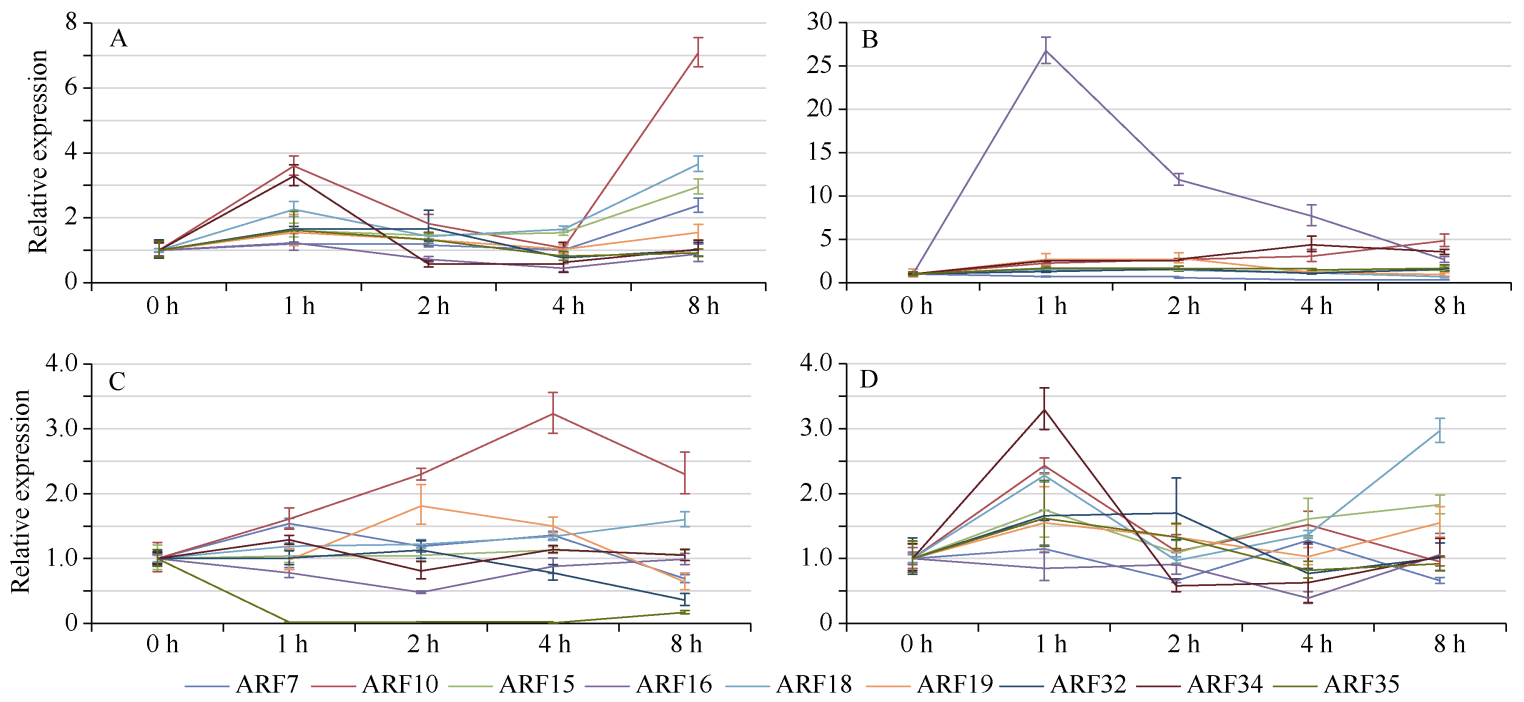

图 3 玉米 $\boldsymbol{A R F}$ 家族基因在不同逆境胁迫下的表达水平

Fig. 3 Transcriptional patterns of $A R F$ genes under different stress treatments

$\mathrm{A}$ : 冷胁迫处理; $\mathrm{B}$ : 热胁迫处理; $\mathrm{C}: \mathrm{NaCl}$ 胁迫处理; $\mathrm{D}$ : 渗透胁迫处理。

A: cold stress treatment; B: hot stress treatment; $\mathrm{C}$ : $\mathrm{NaCl}$ stress treatment; D: osmotic stress treatment.

到的转录组数据和实时定量PCR结果可以看出, Clade C主要在幼制雄花序中优势表达, 而Clade A和Clade B 则主要在幼嫩花序中高水平表达, 有的在幼嫩䧳花序 中优势表达, 有的在雄花序中优势表达。不管 $A R F$ 基因 通过转录激活(Clade A) 还是转录抑制(Clade B), 又或 者通过别的调控途径(Clade C), 都会通过参与调控生 长素相关基因影响玉米花序发育过程。

$A R F$ 基因参与了诸多生物学过程。拟南芥中的 $\operatorname{arf} 2$ 突变体花期、叶衰亡和花脱落时间推迟 ${ }^{[27]}$, 推测 与之同源的玉米 $A R F 10$ 和 $A R F 29$ 也参与花发育和叶 片发育, 而这 2 个基因都在幼嫩花序中优势表达, 也 从侧面印证这一推测; AtARF5 参与胚模式建成和维 管组织形成 ${ }^{[12,28-29]}$, 而作为同源基因的玉米 $A R F 6$ 在 幼嫩花序和中柱中高表达, ARF 33 在幼嫩花序和果 皮中高表达, 这也暗示这 2 个基因可能参与胚发育 和微管形成; AtARF6调控花成熟 ${ }^{[16,30]}$, 同源基因玉 米 $A R F 16$ 和 $A R F 18$ 在幼嫩花序和胚中高表达, 可能 参与花和果穗形成过程; $A t A R F 8$ 是果实起始发育的 负调控因子 ${ }^{[16,30]}$, 黄瓜 $A t A R F 8$ 的同源基因Csa019264 和Csa019265在单性结实和非单性结实品种的表达 差异大 ${ }^{[31]}$, 而玉米AtARF8的同源基因 $A R F 5$ 和 $A R F 34$ 在幼嫩花序和胚中高表达, 推测参与果穗的发育和 形态建成。然而玉米 $A R F$ 基因众多, 通过DAP-seq测 序技术获得的顺式作用元件分析结果显示, $A R F$ 基因 在Clade A、Clade B和Clade C 组间的结合位点特异性 和靶基因等差异不大 ${ }^{[32]}$, 暗示基因功能冗余现象严
重, 这也是至今还未发现有表型的玉米arf突变体的 原因 ${ }^{[25]}$, 开展功能研究还需新的思路和方法。

随着玉米基因组测序完成，高通量测序技术被 广泛应用于基因功能研究。通过分析数据库收录的 测序数据, 可以获得不同部位组织器官在不同发育 时期、不同环境条件下的表达模式。Liu等 ${ }^{[20]}$ 通过 EST数据库预测 $A R F$ 基因主要在雄穗和果穗中表达, Gallavotti等 ${ }^{[26]}$ 通过数据库数据分析 $A R F$ 基因在胚、 幼嫩花序和成熟花序中优势表达, 而本文结合实时 定量PCR 方法和现有数据库更新的转录组数据分析 了玉米 $A R F$ 基因在不同组织器官的表达模式, 发现 $A R F$ 基因主要在雄花序和雌花序中优势表达, 充分 说明 $A R F$ 基因在花序发育过程中发挥重要功能。

$A R F$ 基因在植物的生长发育过程中发挥了重要 作用, 也参与了许多逆境胁迫响应过程。拟南芥 $A R F 5$ 参与维管形成, 而干旱条件下植株的维管合成 能力加强, 暗示 $A R F 5$ 通过参与维管合成而影响植株 的抗旱性 ${ }^{\left[{ }^{[6]}\right.}$; ARF10和 $A R F 16$ 通过控制根冠的发育来 影响植株的抗旱性 ${ }^{[33]}$; 大豆 $A R F$ 基因的表达量在水 分胁迫下发生明显变化, 暗示 $A R F$ 基因参与了大豆 水分胁迫响应 ${ }^{[34]}$ 。玉米中的 $A R F$ 基因在冷、热、盐 和渗透胁迫处理下, 表达量均发生了明显变化, 这 暗示 $A R F$ 基因参与了玉米多种胁迫响应过程。

\section{4 结论}

玉米 $A R F$ 基因家族的 $A R F 10 、 A R F 16$ 和 $A R F 34$ 


\section{呈组成型表达，其余 32 个 $A R F$ 基因均在幼嫩雄花序 和雌花序中高表达, 参与了玉米花序发育过程; 玉 米 $A R F$ 基因家族在冷、热、盐和渗透胁迫处理下表 达量发生明显变化, 暗示玉米 $A R F$ 基因家族参与了 多种逆境胁迫响应过程。}

\section{References}

[1] 吴蓓, 吴建勇, 蔡刘体, 李运合, 黄学林. 生长素反应因子. 植物生理学通讯, 2005, 41: 273-278.

Wu B, Wu J Y, Cai L T, Li Y H, Huang X L. Auxin response factor. Plant Physiol Commun, 2005, 41: 273-278 (in Chinese).

[2] Abel S, Theologis A. Early genes and auxin action. Plant Physiol, 1996, 111: 9 .

[3] Ulmasov T, Hagen G, Guifoyle T J. Dimerization and DNA binding of auxin response factors. Plant J, 1999, 19: 309-319.

[4] Liu Z B, Hagen G, Guifoyle T J. A G-box-binding protein from soybean binds to the E1 auxin-response element in the soybean GH3 promoter and contains a proline-rich repression domain. Plant Physiol, 1997, 115: 397-407.

[5] Wang D, Pei K, Fu Y, Sun Z, Li S, Liu H, Tang K, Han B, Tao Y Z. Genome-wide analysis of the auxin response factors $(A R F)$ gene family in rice (Oryza sativa). Gene, 2007, 394: 13-24.

[6] Singh V K, Rajkumar M S, Garg R, Jain M. Genome-wide identification and co-expression network analysis provide insights into the roles of auxin response factor gene family in chickpea. $S c i$ Rep, 2017, 7: 10895.

[7] Die J V, Gil J, Millan T. Genome-wide identification of the auxin response factor gene family in Cicer arietinum. BMC Genomics, 2018, 19: 301 .

[8] Zhou X, Wu X, Li T, Jia M, Liu X, Zou Y, Liu Z X, Wen F. Identification, characterization, and expression analysis of auxin response factor $(A R F)$ gene family in Brachypodium distachyon. Funct Integr Genomic, 2018, 18: 709-724.

[9] Xu Z, Ji A, Song J, Chen S. Genome-wide analysis of auxin response factor gene family members in medicinal model plant Salvia miltiorrhiza. Biol Open, 2016, 5: 848-857.

[10] Wang S, Hagen G, Guilfoyle T J. ARF-Aux/IAA interactions through domain III/IV are not strictly required for auxin- responsive gene expression. Plant Signal Behav, 2013, 8: e24526.

[11] Remington D L, Vision T J, Guilfoyle T J, Reed J W. Contrasting modes of diversification in the $A u x / I A A$ and $A R F$ gene families. Plant Physiol, 2004, 135: 1738-1752.

[12] Hardtke C S, Berleth T. The Arabidopsis gene MONOPTEROS encodes a transcription factor mediating embryo axis formation and vascular development. EMBO J, 1998, 17: 1405-1411.

[13] Harper R M, Stowe-Evans E L, Luesse D R, Muto H, Tatematsu $\mathrm{K}$, Watahiki M K, Yamamoto K, Liscum E. The NPH4 locus encodes the auxin response factor $A R F 7$, a conditional regulator of differential growth in aerial Arabidopsis tissue. Plant Cell, 2000, 12: 757-770.

[14] Okushima Y, Overvoorde P J, Aeima K, Alonso J M, Chan A, Chang C, Ecker J R, Hughes B. Lui A, Nguyen D. Functional genomic analysis of the AUXIN RESPONSE FACTOR gene family members in Arabidopsis thaliana: unique and overlapping functions of ARF7 and ARF19. Plant Cell, 2005, 17: 444-463.

[15] Wilmoth J C, Wang S, Tiwari S B, Joshi A D, Hagen G, Guifoyle T J, Alonso J M, Ecker J R, Reed J W. NPH4/ARF7 and ARF19 promote leaf expansion and auxin-induced lateral root formation. Plant J, 2005, 43: 118-130.

[16] Nagpal P, Ellis C M, Weber H, Ploense S E, Barkawi L S, Guilfoyle T J, Hagen G, Alonso J M, Cohen J D, Farmer E E. Auxin response factors ARF6 and ARF8 promote jasmonic acid production and flower maturation. Development, 2005, 132: 4107-4118.

[17] Faivre-rampant O, Cardle L, Marshall D, Viola R, Taylor M A. Changes in gene expression during meristem activation processes in Solanum tuberosum with a focus on the regulation of an auxin response factor gene. $J$ Exp Bot, 2004, 55: 613-622.

[18] Waller F, Furuya M, Nick P. OsARF1, an auxin response factor from rice, is auxin-regulated and classifies as a primary auxin responsive gene. Plant Mol Biol, 2002, 50: 415-425.

[19] Jones B, Frasse P, Olmos E, Zegzouti H, Li Z G, Latche A, Pech J C, Bouzayen M. Down-regulation of DR12, an auxin response factor homolog, in the tomato results in a pleiotropic phenotype including dark green and blotchy ripening fruit. Plant J, 2002, 32: 603-613.

[20] Liu Y, Jiang H Y, Chen W J, Qian Y X, Ma Q, Cheng B J, Zhu S W. Genome-wide analysis of the auxin response factor $(A R F)$ gene family in maize (Zea mays). Plant Growth Regul, 2011, 63: 225-234.

[21] Grennan A K. Genevestigator. Facilitating web-based gene expression analysis. Plant Physiol, 2006, 141: 1164-1166.

[22] Lescot M, Dehais P G, Marchal K, Moreau Y, Peer Y V, Rouze P, Rombauts S. PlantCARE, a database of plant cis-acting regulatory elements and a portal to tools for in silico analysis of promoter sequences. Nucl Acids Res, 2002, 30: 325-327.

[23] Galli M, Liu Q, Moss B L, Malcomber S, Li W, Gaines C, Federici S, Roshkovan J, Meeley R, Nemhauser J L, Gallavotti A. Auxin signaling modules regulate maize inflorescence architecture. Proc Natl Acad Sci USA, 2015, 112: 13372-13377.

[24] Xing H Y, Pudake R N, Guo G G, Xing G F, Hu Z R, Zhang Y R, Sun Q X, Ni Z F. Genome-wide identification and expression profiling of auxin response factor $(A R F)$ gene family in maize. BMC Genomics, 2011, 12: 178.

[25] Ulmasov T, Hagen G, Guilfoyle T J. Activation and repression of transcription by auxin response factors. Proc Natl Acad Sci USA, 1999, 96: 5844-5849.

[26] Matthes M S, Best N B, Robil J M, Malcomber S, Gallavotti A, Mcsteen P. Auxin evodevo: conservation and diversification of genes regulating auxin biosynthesis, transport, and signaling. Mol Plant, 2019, 12: 298-320.

[27] Liu Z, Miao L, Huo R, Song X, Johnson C, Kong L, Sundaresan V, Yu X L. ARF2-ARF4 and ARF5 are essential for female and male gametophyte development in Arabidopsis. Plant Cell Physiol, 2018, 59: 179-189.

[28] Hardtke C S, Berleth T. The Arabidopsis gene MONOPTEROS encodes a transcription factor mediating embryo axis formation and vascular development. EMBO J, 1998, 17: 1405-1411.

[29] Wenzel C L, Schuetz M, Yu Q, Mattsson J. Dynamics of MONOPTEROS and PIN-FORMED1 expression during leaf vein pattern formation in Arabidopsis thaliana. Plant J, 2007, 49: 
387-398.

[30] Wu M F, Tian Q, Reed J W. Arabidopsis microRNA167 controls patterns of ARF6 and ARF8 expression, and regulates both female and male reproduction. Development, 2006, 133: 4211-4218.

[31] 王垒, 陈劲枫, 娄丽娜, 娄群峰. 黄瓜 ARF 家族序列特征及部 分成员在果实发育早期的表达分析. 园艺学报, 2011，4: 717-724.

Wang L, Chen J F, Lou L N, Lou Q F. Sequence characteristics of $A R F$ gene family of cucumber and an expression analysis of some members during early development of fruits. Acta Hortic Sin, 2011, 4: 717-724 (in Chinese with English abstract).

[32] Galli M, Khakhar A, Lu Z, Chen Z, Sen S, Joshi T, Nemhauser J L, Schmitz R J, Gallavotti A. The DNA binding landscape of the maize AUXIN RESPONSE FACTOR family. Nat Commun, 2018,
9: 4526.

[33] 王佳伟, 蔡文娟, 王凌健, 毛颖波, 陈晓亚. 生长素响应因子 $A R F 10$ 和 16 控制拟南芥根冠发育. 见: 中国遗传学会植物遗传与 基因组学专业委员会 2005 年学术研讨会论文摘要集, 2005.p 15. Wang J W, Cai W J, Wang L J, Mao Y B, Chen X Y. Auxin response factors $A R F 10$ and 16 control root crown development in Arabidopsis thaliana. In: Genetics Society of China in plant Genetics and Genomics Professional Committee Conference Abstract Set, 2005. p 15 (in Chinses).

[34] Ha C V, Le D T, Nishiyama R, Watanabe Y, Sulieman S, Tran U T, Mochida K, Dong N V, Yamaguchi-Shinozaki K, Shinozaki K, Tran L S. The auxin response factor transcription factor family in soybean: genome-wide identification and expression analyses during development and water stress. DNA Res, 2013, 20: 511-524. 